\title{
Effect of molecular architecture of copolymer template on the morphology of mesoporous methylsilsesquioxane
}

\author{
Chia-Hua Lee ${ }^{\text {a }}$, Wen-Chang Chen ${ }^{\text {a,b,*, Jen-Yung Hsu }}{ }^{c}$, Hsin-Lung Chen ${ }^{\text {c,*** }}$ \\ a Institute of Polymer Science and Engineering, National Taiwan University, Taipei 10617, Taiwan \\ ${ }^{\mathrm{b}}$ Department of Chemical Engineering, National Taiwan University, Taipei 10617, Taiwan \\ ${ }^{\mathrm{c}}$ Department of Chemical Engineering, National Tsing-Hua University, Hsin-Chu 30013, Taiwan
}

Received 23 November 2006; received in revised form 20 April 2007; accepted 20 April 2007

Available online 4 May 2007

\begin{abstract}
Hybrid and mesoporous materials derived from methylsilsesquixoane (MSSQ) have been prepared by the evaporation-induced self-assembly (EISA) process using polystyrene-poly(2-vinyl pyridine) (PS-P2VP) block copolymers with linear (LA) and hetero-arm star (HA) molecular architectures as the templates. TEM and SAXS were used to characterize the morphology of the hybrid materials, LA/MSSQ (LB) and HA/ MSSQ (HB), and their corresponding porous materials, LP and HP, obtained by pyrolysis. TEM images suggested that the morphology transformed from random spheres in LB hybrid to cylinders in the corresponding LP porous material. However, the sphere morphology was effectively preserved after the pyrolysis of HB. We proposed that MSSQ initially solubilized into the P2VP coronas of the copolymer micelles tended to be excluded from the coronal regions during the extensive curing of MSSQ on heating to the calcination temperature. This dewetting process competed against the crosslinking of MSSQ that could freeze the structure if the network formed readily prior to the abundant exclusion of MSSQ. In the hetero-arm system where several pairs of copolymer chains jointed to a common core, the exclusion of the MSSQ out of the corona regions became more restricted. Consequently, the initial micelle morphology was effectively fixed by the crosslinking reaction. The present study demonstrated that the molecular architecture of the structure-directing agent played an important role in the morphological formation of the MSSQ-based mesoporous materials.
\end{abstract}

(C) 2007 Elsevier Ltd. All rights reserved.

Keywords: Molecular architecture; Block copolymer; Porous

\section{Introduction}

Porous materials have shown potential applications in catalysis, adsorption, electronics, optoelectronics, drug delivery and ultralight structural materials [1-7]. One of the general methodologies to generate nanopores in inorganic oxides is through the templating process. This approach takes the advantage of self-assembly in controlling the pore shape, pore

\footnotetext{
* Corresponding author. Department of Chemical Engineering, National Taiwan University, No. 1, Sec. 4, Roosevelt Rd., Taipei 10617, Taiwan. Tel.: +886 23628398; fax: +886223623040.

** Corresponding author.

E-mail addresses: chenwc@ntu.edu.tw (W.-C. Chen), hslchen@mx.nthu. edu.tw (H.-L. Chen).
}

size distribution and structural regularity. For instance, the "evaporation-induced self-assembly" (EISA) [8,9] combined with the templating process of the structure-directing agents can lead to well-ordered porous materials with pore dimensions ranging from 2 to $50 \mathrm{~nm}[9-16]$.

For the preparation of mesoporous silica materials using the EISA process, the silica precursor is first mixed with the structure-directing agent to form a homogeneous solution. Then, the solvent is allowed to evaporate slowly to induce the self-assembly of the structure-directing agent in the silica matrix. A calcination process for removing the organic components in the hybrid then follows to produce the mesoporous material. Amphiphilic block copolymers (ABCs) such as polystyrene-block-poly(ethylene oxide) (PS- $b$-PEO), poly(ethylene oxide)-block-poly(propylene oxide)-block-poly(ethylene oxide) 


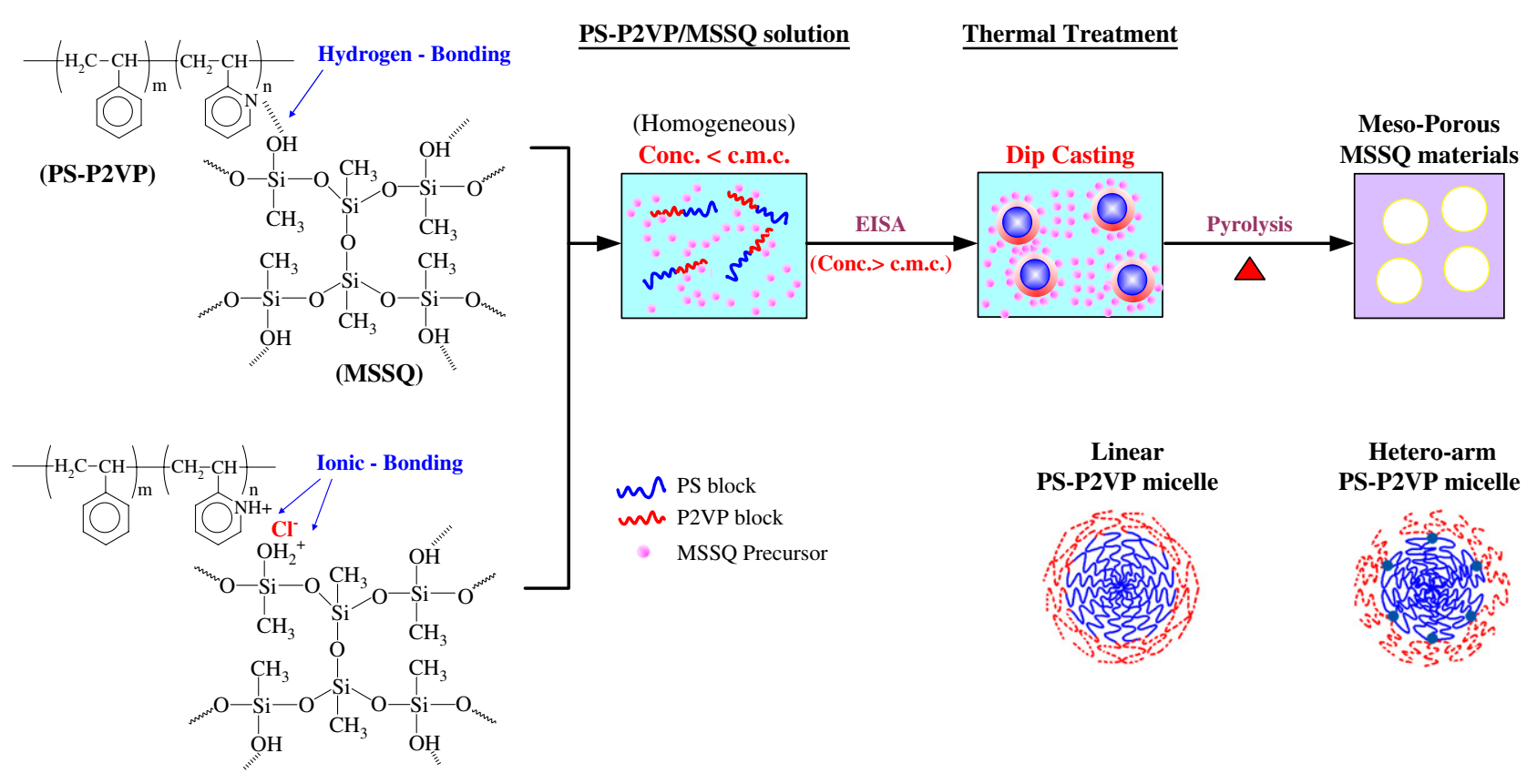

Fig. 1. Schematic illustration showing the procedure for preparing the mesoporous MSSQ material using block copolymer as the template.

(PEO- $b$-PPO- $b$-PEO) [12-16] have been widely used as the structure-directing agents in the EISA process. The physical interaction such as hydrogen bonding and ionic bonding between the inorganic precursor and the $\mathrm{ABC}$ is considered as an important factor for controlling the pore size distribution of the resultant mesoporous materials. Furthermore, a slow curing process with an optimum temperature control is necessary to minimize the structure deformation as the $A B C$ is degraded from the hybrid materials.

Previous studies on the preparations of mesoporous structures using $\mathrm{ABCs}$ as the structure-directing agents have been based primarily on the block copolymers with linear architecture. With the advancement of synthetic techniques, the copolymers with more complex architectures such as hetero-arm and block-arm stars can now be synthesized. It has been shown that these copolymers self-assemble in essentially the same way as their linear counterpart. However, the architectural complexity results in much slower phase separation kinetics and modification of the equilibrium structures [17-20]. In the block-arm star copolymers, the tendency of the core block to locate inside the cylindrical and spherical microdomains leads to an asymmetry in the phase diagram whereas in the case of hetero-arm star copolymers the difference in the number of arms of the two blocks causes an asymmetry in the phase diagram compared to that of the corresponding linear diblock.

In this study, we attempt to reveal the effect of molecular architecture of the copolymer template on the mesoporous structures generated through the EISA process. Here we used polystyrene-poly(2-vinylpyridine) (PS-P2VP) block copolymers with linear (denoted by LA) and hetero-arm star (denoted by HA) architectures as the structure-directing agents for preparing the hybrids and mesoporous materials from methylsilsesquioxane (MSSQ) using EISA. The methodology adopted here is schematically illustrated in Fig. 1. First, a homogeneous solution of the MSSQ precursor and the structuredirecting agent, LA or HA, was prepared, in which the concentration of LA or HA was lower than the critical micelle concentration (c.m.c.). The miscibility between the copolymer and MSSQ in the solution was promoted by the hydrogen bonding or the ionic bonding between MSSQ precursor and P2VP blocks. Then, the solvent was allowed to evaporate slowly at room temperature. During the solvent evaporation the block copolymer underwent self-assembly above the c.m.c., generating a hybrid material containing micelles with PS cores and P2VP coronas dispersed in the MSSQ precursor matrix. The system was subsequently heated slowly to the calcination temperature to remove the organic components to yield the mesoporous material. During the heating the MSSQ precursor underwent crosslinking reaction. If this curing process was rapid, the morphology of the copolymer micelles could be kinetically frozen by the highly viscous crosslinked MSSQ matrix, thereby suppressing the macrophase separation and any structure reorganization induced by the curing reaction. In this case, the micelles of the copolymer template could properly prescribe the pore morphology of the resultant mesoporous material.

In this study, we investigate the morphology of the hybrids (i.e., the samples having been cured thermally but without degradation of the organic components) and the mesoporous MSSQ materials derived from LA and HA by means of transmission electron microscopy (TEM) and small angle $\mathrm{X}$-ray scattering (SAXS) to reveal the molecular architecture effect of the copolymer template on the structure of the MSSQ materials. It will be shown that HA acted as a more effective template than LA because of its stronger resistance to the morphological perturbation induced by the MSSQ curing. 


\section{Experimental section}

\subsection{Materials}

Styrene (St, Fluka, 99\%), 2-vinylpyridine (2VP, Acros, 97\%), and divinylbenzene (DVB, Aldrich, 80\%) were purchased and distillated from dibutylmagnesium (Aldrich, $1.0 \mathrm{M}$ solution in heptane) under reduced pressure. Tetrahydrofuran (THF, Acros, 99.9\%) was distillated from sodium (Aldrich, 30-35 wt \% dispersion in paraffin wax) and benzophenone (Aldrich, 99+\%) mixture. Acetone (Acros, 99+\%), hydrochloric acid $(\mathrm{HCl}$, Acros, 37\%), sec-butyllithium (sec$\mathrm{BuLi}$, Acros, 1.3 M in cyclohexane/hexane), and methyl trimethoxysilane (MTMS, Aldrich, 95\%) were used as received.

\subsection{Synthesis of $P S-P 2 V P$ copolymers}

\subsubsection{Synthesis of linear PS-b-P2VP block copolymer}

Linear PS- $b$-P2VP was synthesized by sequential living anionic polymerization [21], as shown in Fig. 2(a). The polymerization was carried out in THF at $-78^{\circ} \mathrm{C}$ using sec-BuLi as the initiator. The polymerization was terminated by an aliquot of methanol and the polymer product was precipitated in hexane followed by purification by fractionation. The linear diblock copolymer denoted by LA had the weight-average molecular weight $\left(M_{\mathrm{w}}\right)$ of 23,200 and a polydispersity index (PDI) of 1.12. The volume fraction of PS $\left(f_{\mathrm{PS}}\right)$ in the neat copolymer was 0.70 .

\subsubsection{Synthesis of hetero-arm star $(P S)_{n}(P 2 V P)_{n}$ block copolymer}

A three-step sequential copolymerization was used to prepare $\mathrm{A}_{n} \mathrm{~B}_{n} \mathrm{PS}-\mathrm{P} 2 \mathrm{VP}$ hetero-arm star copolymer as illustrated

(a)

St

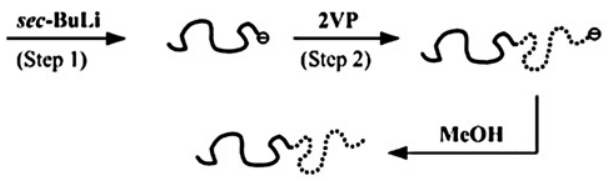

(b)

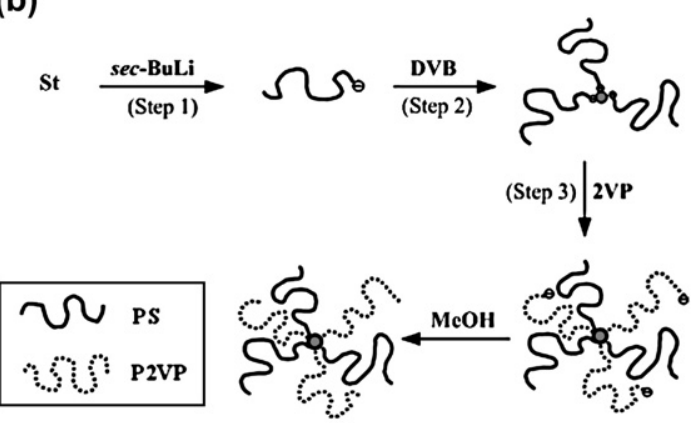

Fig. 2. Schematic illustrations of the syntheses of (a) linear PS-P2VP; (b) hetero-arm star copolymer, PS-P2VP, by sequential living anionic polymerization.
Table 1

Molecular characteristics of the prepared polystyrene-poly(2-vinylpyridine) copolymers

\begin{tabular}{lllllll}
\hline Sample & $M_{\mathrm{w}}{ }^{\mathrm{a}}$ overall & ${M_{\mathrm{w}}}^{\mathrm{b}}$ PS-arm & $M_{\mathrm{w}}{ }^{\mathrm{c}}$ P2VP-arm & $M_{\mathrm{w}} / M_{\mathrm{n}}$ & $N^{\mathrm{d}}$ & $f_{\mathrm{PS}}{ }^{\mathrm{e}}$ \\
\hline LA & 23,200 & 16,200 & 7000 & 1.12 & - & 0.70 \\
HA & 65,900 & 15,600 & 7500 & 1.21 & 5.7 & 0.67 \\
\hline
\end{tabular}

${ }^{\mathrm{a}}$ Weight-average molecular weight of the whole copolymer.

${ }^{b}$ Weight-average molecular weight of PS block.

${ }^{c}$ Weight-average molecular weight of P2VP block.

d Average number of PS and P2VP arms in the star copolymer.

${ }^{\mathrm{e}}$ Overall volume fraction of PS in the neat copolymer.

in Fig. 2(b). The synthetic scheme was similar to that of P2VPpoly(tert-butyl acrylate) hetero-arm star copolymer reported in the literature $[22,23]$. First some living PS arms were synthesized at $-78^{\circ} \mathrm{C}$ using sec-BuLi as the initiator. These PS arms were then joined together in the second step by reacting the living PS chains with a small amount of divinylbenzene (DVB). A star-shaped (PS) ${ }_{n}$ was thus formed, bearing a number of active sites at the DVB core which was equal to the number of the attached PS arms. In the third step, a second generation of P2VP arms grew from the cores on adding 2vinylpyridine (2VP) to the reaction mixture. The living anionic polymerization was terminated by methanol, precipitated in hexane, and then filtered out to obtain the polymer product. The product was subsequently purified by extraction using cyclohexane/heptane (V/V, 95/5) followed by fractionation by GPC to remove the unattached PS arms present due to accidental deactivation during the DVB polymerization.

The copolymer obtained after the purification had a $M_{\mathrm{w}}$ of 65,900 with a PDI of 1.21, and the volume fraction of PS was 0.67 . The average number of arms in the hetero-arm star copolymer was 5.7 ; therefore the copolymer was denoted as $\mathrm{PS}_{3} \mathrm{P}_{2} \mathrm{VP}_{3}$ and henceforth will be referred as HA. The detailed molecular characteristics of $\mathbf{H A}$ and $\mathbf{L A}$ are given in Table 1. Both materials had approximately the same $f_{\mathrm{PS}}$ and block lengths.

\subsection{Synthesis of methylsilsesquioxane (MSSQ)}

The synthesis and characterization of MSSQ followed the procedure reported previously [24-26]. MSSQ precursors were synthesized from methyl trimethoxysilane (MTMS) in THF by sol-gel process. The number average molecular weight of the prepared MSSQ determined by GPC was 953 and the PDI was 1.83 , which suggested the oligomeric characteristics of the prepared MSSQ. The chemical structure of the resultant MSSQ precursors was characterized by FTIR. The absorption peaks observed at 906, 1030 , and $1120 \mathrm{~cm}^{-1}$ were assigned as $\mathrm{Si}-\mathrm{OH}$ stretching, $\mathrm{Si}-\mathrm{O}-\mathrm{Si}$ stretching of non-symmetric (network-like) structure, and $\mathrm{Si}-\mathrm{O}-\mathrm{Si}$ stretching of symmetric (cage-like) structure, respectively. The prepared MSSQ precursors contained $\mathrm{Si}-\mathrm{OH}$ groups due to the incomplete condensation. Comparing with a reference sample from Gelest Co. with a reported $\mathrm{OH}$ content of 5\%, the content of the $\mathrm{SiOH}$ moiety in the MSSQ precursors prepared here was approximately $12.1 \%$. 


\subsection{Preparation of the mesoporous materials using $\mathbf{L A}$ and $\boldsymbol{H A}$ as templates}

Fig. 1 illustrates the preparation procedure of mesoporous MSSQ materials. The block polymer was first dissolved in THF to obtain a homogeneous solution followed by the addition of MSSQ solution. The weight ratio of the copolymer to MSSQ was 40:60. The solution was dried in air at ambient temperature for 1 day and subsequently placed in a vacuum oven at $40{ }^{\circ} \mathrm{C}$ for $30 \mathrm{~min}$ and then $65^{\circ} \mathrm{C}$ for overnight. The resultant micellar solution was further dried at 80 and $100{ }^{\circ} \mathrm{C}$ sequentially for $30 \mathrm{~min}$ followed by baking at $120^{\circ} \mathrm{C}$ for overnight. MSSQ underwent some extent of crosslinking reaction during this baking. The organic/inorganic hybrid materials, LA/MSSQ and HA/MSSQ, thus obtained were referred to as $\mathbf{L B}$ and $\mathbf{H B}$, respectively. These hybrid materials were subsequently pyrolyzed in a quartz furnace under nitrogen with heating from 120 to $400{ }^{\circ} \mathrm{C}$ at $5{ }^{\circ} \mathrm{C} / \mathrm{min}$. Further crosslinking reaction took place extensively before the intervention of thermal degradation during this heating process. After reaching $400{ }^{\circ} \mathrm{C}$ the sample was allowed to stay there for $3 \mathrm{~h}$ to remove the copolymer species completely. As a result, the mesoporous materials using LA and HA as the templates were obtained and were denoted by $\mathbf{L P}$ and HP, respectively.

\subsection{Characterizations}

The FTIR spectra of the prepared materials on $\mathrm{KBr}$ pellets were recorded by a DIGILAB FTS-3500GX spectrophotometer with the resolution of $2 \mathrm{~cm}^{-1}$. The molecular weights of the prepared materials were determined by a gel permeation chromatography (GPC) equipped with PLgel $5 \mu \mathrm{m}$ MIXED-C and D columns, an RI detector, and the elution solvent of THF at $1 \mathrm{ml} / \mathrm{min}$. The TEM images of the prepared hybrid and mesoporous materials were obtained from a JEOLJEM2000-FXZ transmission electron microscopy with an accelerating voltage of $100 \mathrm{kV}$. SAXS measurements were performed using a Bruker Nanostar SAXS instrument. The X-ray source, a $1.5 \mathrm{~kW}$ X-ray ( $\mathrm{Cu} \mathrm{K} \alpha$ radiation $\lambda=1.54 \AA$ ) generator (Kristalloflex 760) equipped with a $\mathrm{Cu}$ tube, was operated at $35 \mathrm{~mA}$ and $40 \mathrm{kV}$. The scattering intensity was detected by a two-dimensional position-sensitive detector (Bruker AXS) with $512 \times 512$ channels. The area scattering pattern had been radially averaged to increase the photon counting efficiency compared with the one-dimensional linear detector. The intensity profile was expressed as the plot of the scattering intensity $(I)$ vs. the scattering vector, $q=4 \pi / \lambda \sin (\theta / 2)(\theta=$ scattering angle). All scattering data were corrected by the empty beam scattering and the sensitivity of each pixel of the area detector.

\section{Results and discussion}

\subsection{Analysis of the physical bonding between PS-P2VP and MSSQ}

Fig. 3 shows the FTIR spectra of the prepared MSSQ precursor and their hybrid materials, $\mathbf{L B}$ and $\mathbf{H B}$, in the

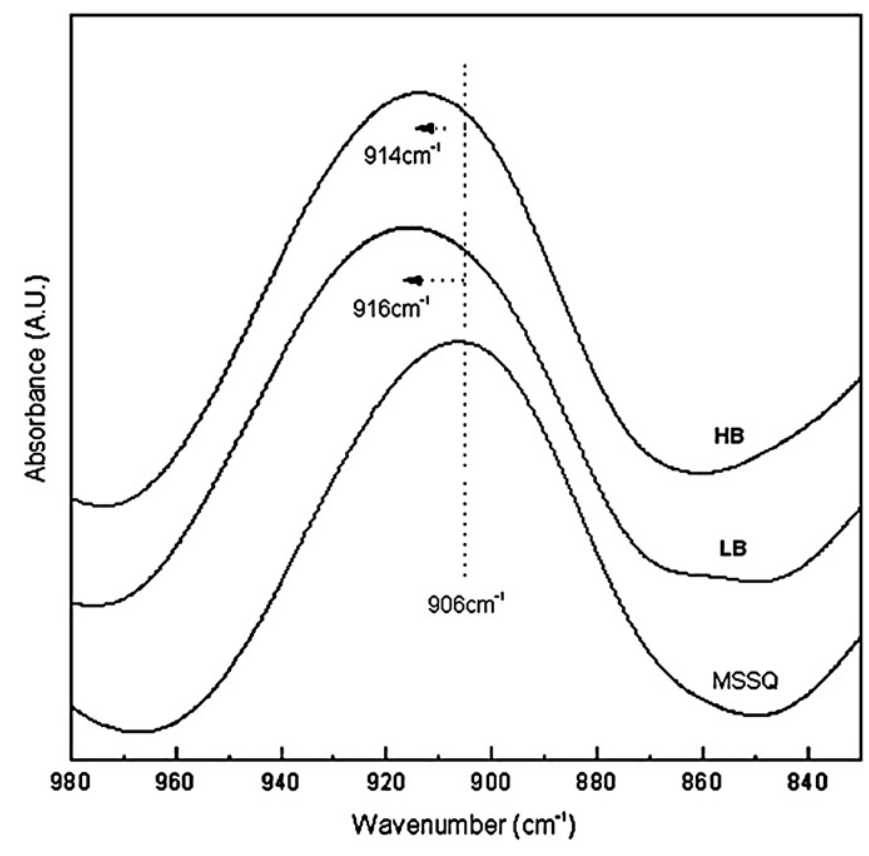

Fig. 3. FTIR spectra of MSSQ, $\mathbf{L B}$, and $\mathbf{H B}$ in the wavenumber range of $820-980 \mathrm{~cm}^{-1}$.

wavenumber region of $820-980 \mathrm{~cm}^{-1}$. The peak at $906 \mathrm{~cm}^{-1}$ associated with the $\mathrm{Si}-\mathrm{O}$ stretching of the $\mathrm{Si}-\mathrm{OH}$ group of MSSQ shifted to the higher wavenumber of 916 and $914 \mathrm{~cm}^{-1}$ for $\mathbf{L B}$ and $\mathbf{H B}$, respectively, indicating that hydrogen bonding existed between the hydroxyl groups in MSSQ and the pyridine moiety of P2VP before crosslinking. Moreover, the $\mathrm{H}^{+}$ion residue from the $\mathrm{HCl}$ catalyst for preparing MSSQ might transfer to $\mathrm{SiOH}$ and pyridine groups to produce the ionic species $\mathrm{SiOH}_{2}^{+}$and $\mathrm{NH}^{+}$, respectively, for providing ionic bondings. The $\mathrm{Si}-\mathrm{O}$ bond strength was increased by the formation of hydrogen bonding and ionic bonding, such that the $\mathrm{Si}-\mathrm{O}$ stretching frequency increased accordingly. These strong intermolecular interactions enhanced the miscibility between MSSQ precursor and the copolymers and hence prevented large-scale phase separation before thermal curing.

\subsection{Morphologies of the hybrids and their corresponding porous materials}

Fig. 4(a) shows the TEM micrograph of the unstained hybrid material LB and Fig. 4(b) displays the image obtained by tilting the specimen in (a) by $20^{\circ}$. The bright regions in the micrograph correspond to the domains formed by the LA copolymer while the dark region is the MSSQ matrix with a higher electron density due to the presence of Si element. Since the image obtained by tilting the specimen by $20^{\circ}$ (Fig. 4(b)) was not significantly different from that shown in Fig. 4(a), the TEM observation indicated that LB hybrid exhibited a random sphere morphology where the spherical copolymer domains of around $25 \mathrm{~nm}$ in diameter arranged in a liquid-like disorder. The spheres were found to transform into cylinders in the corresponding mesoporous material, $\mathbf{L P}$, 

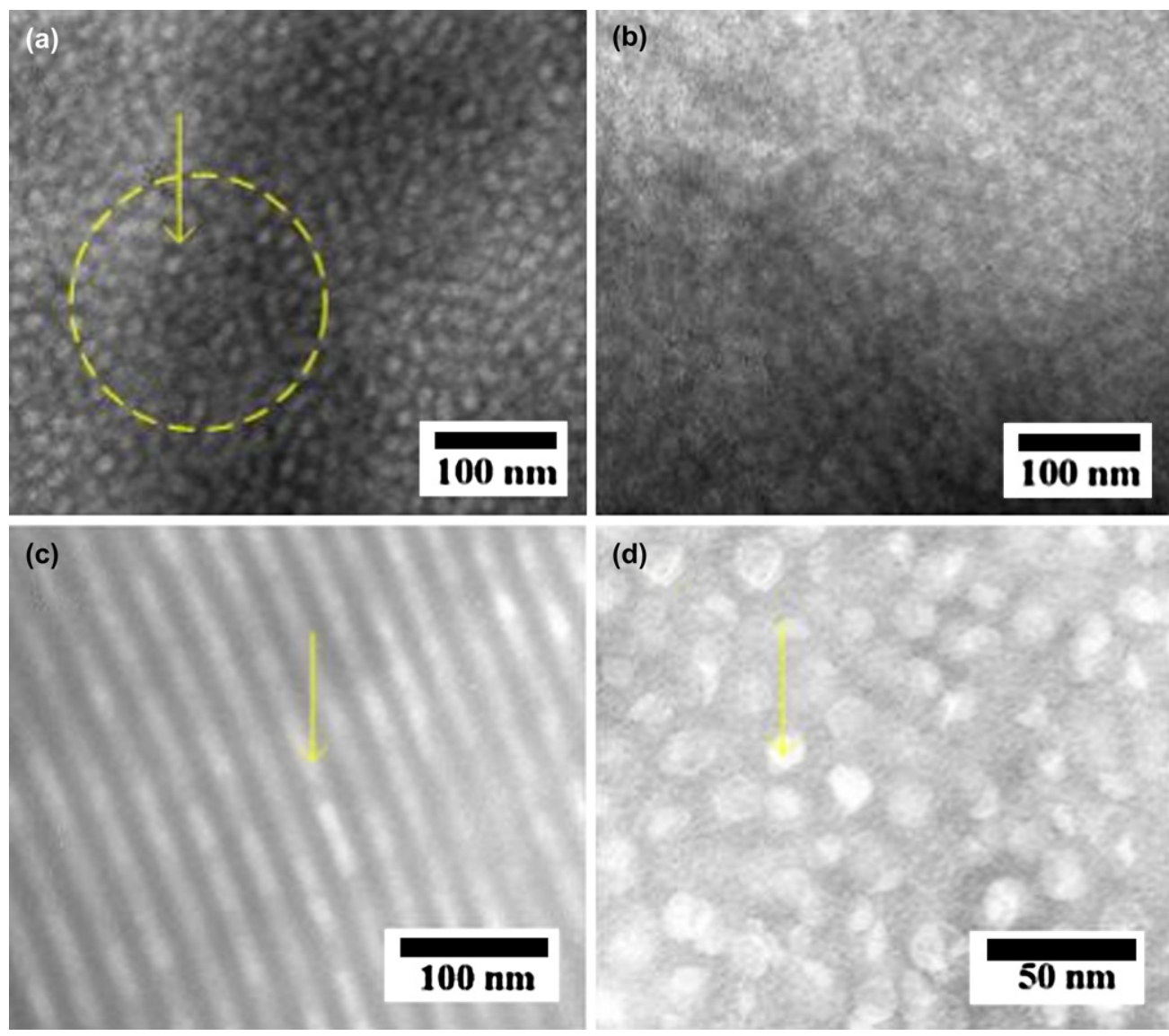

(d)

Fig. 4. TEM images of (a) $\mathbf{L B}$, (b) the $\mathbf{L B}$ specimen of (a) tilted by $20^{\circ}$, (c) the side view of the cylindrical pores in $\mathbf{L P}$ and (d) the top view of the cylindrical pores in $\mathbf{L P}$.

as demonstrated by the TEM micrographs in Fig. 4(c) and (d) showing the two types of images observed simultaneously on a LP specimen. The striation pattern in Fig. 4(c) corresponded to the side view of the cylindrical pores, while the image in Fig. 4(d) represented the top view of the cylinders without apparent long-range order. Consequently, the heating of $\mathbf{L B}$ to the calcination temperature (i.e., from 120 to $400{ }^{\circ} \mathrm{C}$ at $5^{\circ} \mathrm{C} / \mathrm{min}$ ), which induced extensive curing of MSSQ prior to the thermal degradation of the copolymer, disrupted the original morphology of the copolymer domains and transformed it to random cylinders.

Interestingly, the morphological transformation was not observed in the hetero-arm system. Fig. 5(a) displays the TEM micrograph of the HB hybrid and Fig. 5(b) corresponds to the image obtained by tilting the specimen in (a) by $20^{\circ}$. HB also exhibited a sphere morphology but in contrast to $\mathbf{L B}$, the spherical domains were found to pack in an ordered lattice. This long-range ordered sphere morphology was effectively preserved after the pyrolysis, as can be seen from Fig. 5(c) and (d) showing the only type of image observed for the corresponding mesoporous material, HP. Assuming that the spherical domains and pores are arranged in the most common body-centered cubic (BCC) lattice for block copolymers, then the image in Fig. 5(a) and (c) would correspond to the projections of the lattice in $\{100\}$ and (110) directions, respectively. The sphere diameter $(D)$ and the inter-domain distance $(d)$ estimated from the assumptions of these projections were ca. 23.5 and $33.1 \mathrm{~nm}$, respectively, for $\mathbf{H B}$ and 23.5 and $34.3 \mathrm{~nm}$ for HP. The observed domain sizes and inter-domain distances appeared to be larger than those reported for PS- $b$-P2VP with similar molecular weight [27]. This may be attributed to the presence of MSSQ which perturbed the aggregation number of the PS microdomains. Moreover, the nonequilibrium factors associated with the crosslinking reaction of MSSQ were also expected to influence the domain size and the inter-domain distance. With the knowledge of $D$ and $d$ the pore volume fraction $\phi$ of HP could be obtained by Eq. (1) [28]

$\phi=\frac{\sqrt{3} \pi}{8}\left(\frac{D}{d}\right)^{3}$

The value of $\phi$ thus calculated was 0.22 . This value was in reasonable agreement with the volume fraction of PS $(\sim 0.27)$ in the hybrid.

The morphologies of the hybrids and the mesoporous materials were also characterized by SAXS to yield more accurate morphological parameters associated with the mesostructures. Fig. 6 shows the SAXS profiles of LB and LP. The scattering profiles were characterized by a primary scattering peak at 0.21 and $0.24 \mathrm{~nm}^{-1}$ for $\mathbf{L B}$ and $\mathbf{L P}$, respectively, along with a shallow hump (pinpointed by " $i=1$ ") corresponding to the first-order form factor peak associated with the scattering 

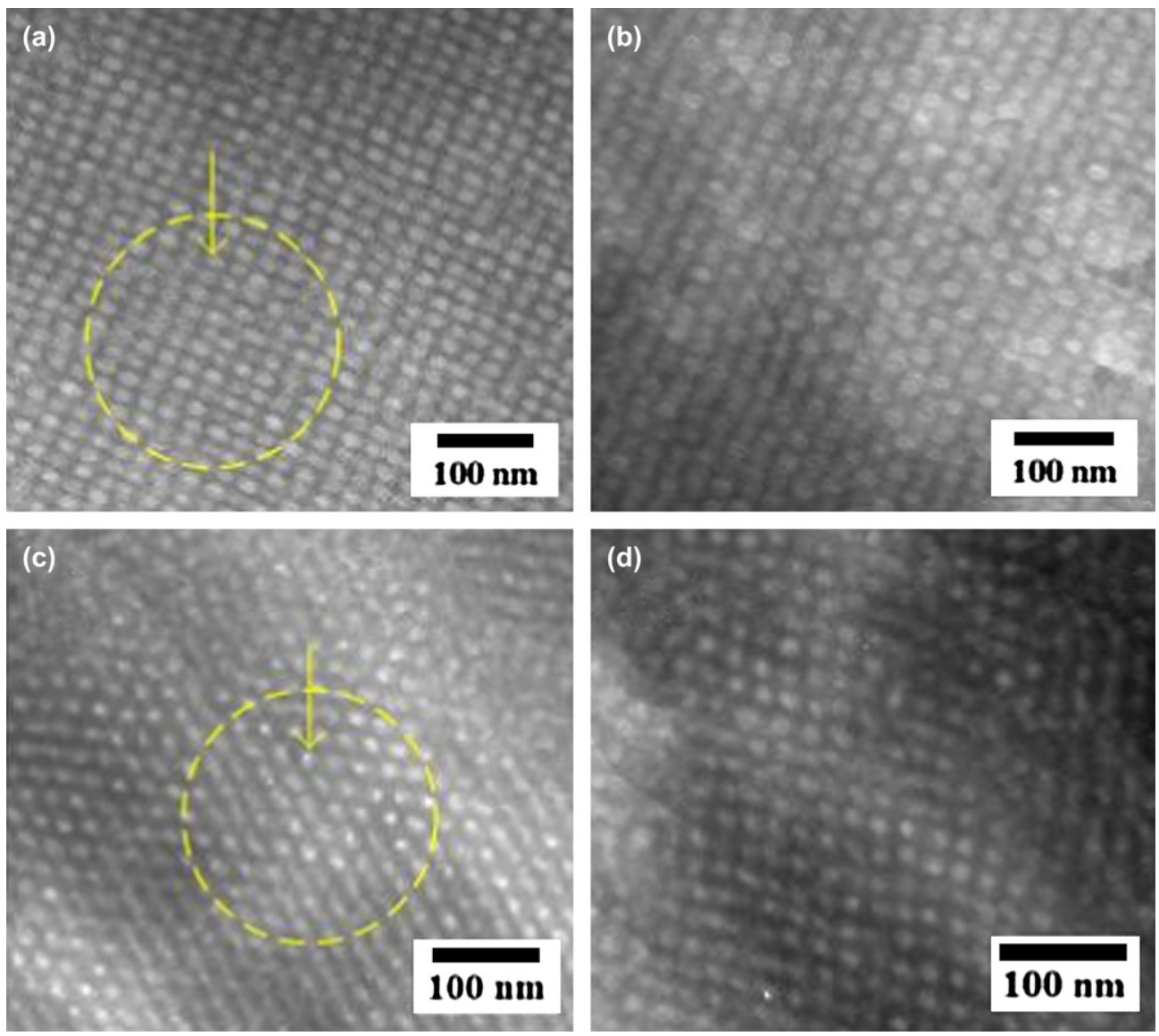

Fig. 5. TEM images of (a) $\mathbf{H B}$, (b) the HB specimen of (a) tilted by $20^{\circ}$, (c) HP and (d) the HP specimen of (c) tilted by $20^{\circ}$.

from the individual domains or pores. The form factor peak was hardly discernible in the profile of $\mathbf{L P}$, suggesting that the domain structure was disturbed by the pyrolysis. The scattering intensity of $\mathbf{L P}$ was considerably higher than that of $\mathbf{L B}$ due to the much larger electron density contrast between the pores and the matrix.

Fig. 7 displays the SAXS profiles of $\mathbf{H B}$ and HP. The primary peak of the hybrid was found to locate at $0.23 \mathrm{~nm}^{-1}$. In contrast to the linear template where the primary peak shifted to higher $q$ after pyrolysis, the position of the primary peak of $\mathbf{L P}$ remained at $0.23 \mathrm{~nm}^{-1}$. This confirmed that the hetero-arm star copolymer exhibited a strong resistance to structural perturbation induced by the crosslinking reaction. It should be noted that neither HB nor HP showed clear higher-order lattice peaks in the SAXS profiles, which indicated that the grains containing the ordered domains or pores ( $c f$. Fig. 5) were small. The inter-domian distance in HP calculated from the primary peak position $\left(q_{1}\right)$ assuming BCC lattice was $d=(3 / 2)^{1 / 2} 2 \pi / q_{1}=33.5 \mathrm{~nm}$, closely agreed with that deduced from the TEM image.

The SAXS profile of $\mathbf{H P}$ was further analyzed to obtain the average pore size and the pore volume fraction. The form factor of a sphere with radius of $R$ is known to display a series of maximum at $q R=5.765,9.095$. Thus the average radius of the spherical pores in HP was obtained from the position of the first form factor peak $\left(q_{i=1}\right)$ via $R=5.765 / q_{i=1} \approx 12 \mathrm{~nm}$.
This value also agreed with that estimated from the TEM image. The pore volume fraction calculated from the assumption of BCC lattice using the values of $d$ and $R$ was 0.25 .

The average pore size may also be obtained from the threedimensional correlation function obtained from the Fourier transform of the scattering intensity, viz. Eq. (2) [29].

$\gamma_{3}(r)=\frac{1}{Q}\left\{\int_{0}^{\infty} I(q) q^{2} \frac{\sin (q r)}{q r} \mathrm{~d} q\right\}$

where $Q$ is the scattering invariant given by

$Q=\int_{0}^{\infty} I(q) q^{2} \mathrm{~d} q$

Fig. 8 shows the three-dimensional correlation function of $\mathbf{H P .}$ For an ideal two-phase model, $\gamma_{3}(r)$ at small $r$ is given by Eq. (4) [29]

$\gamma_{3}(r) \cong 1-\frac{r}{l \mathrm{p}}$

where $l_{\mathrm{p}}$ is the Porod length given by Eq. (5) [29] 


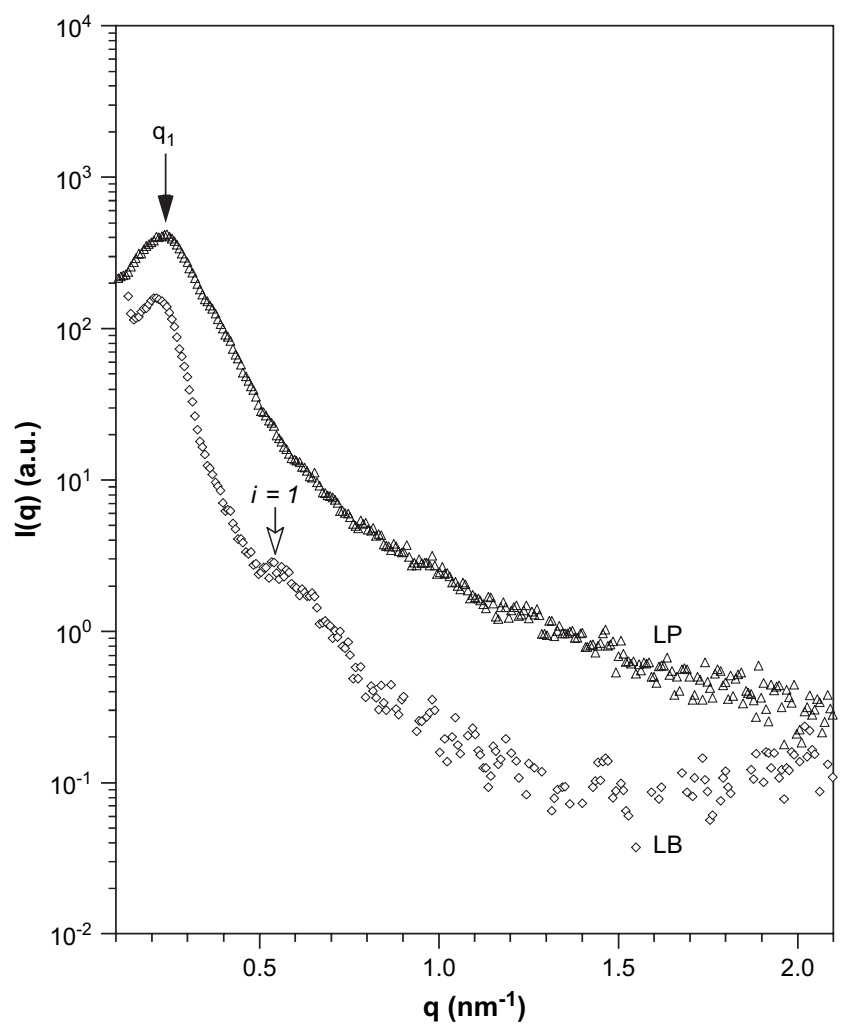

Fig. 6. SAXS profiles of the $\mathbf{L B}$ and $\mathbf{L P}$. The first form factor peak is denoted by " $i=1$ ".

$\frac{1}{l_{\mathrm{p}}}=\frac{1}{4 \phi(1-\phi)}\left(\frac{S}{V}\right)$

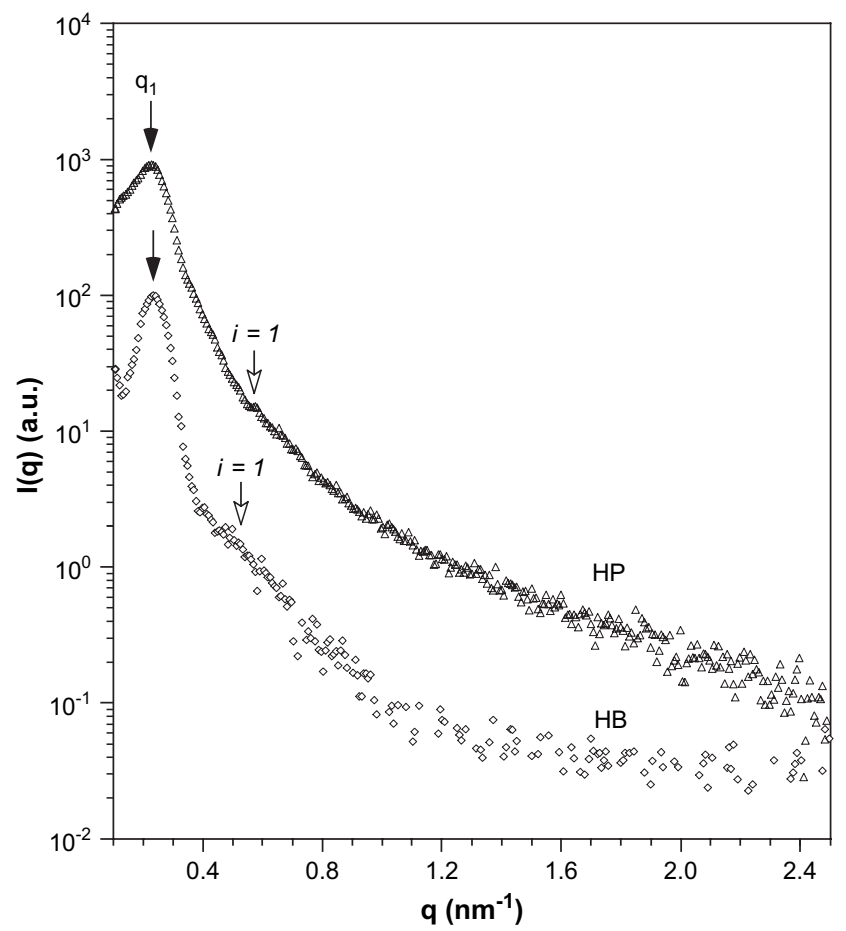

Fig. 7. SAXS profiles of the HB and HP. The first form factor peak is denoted by " $i=1$ ".

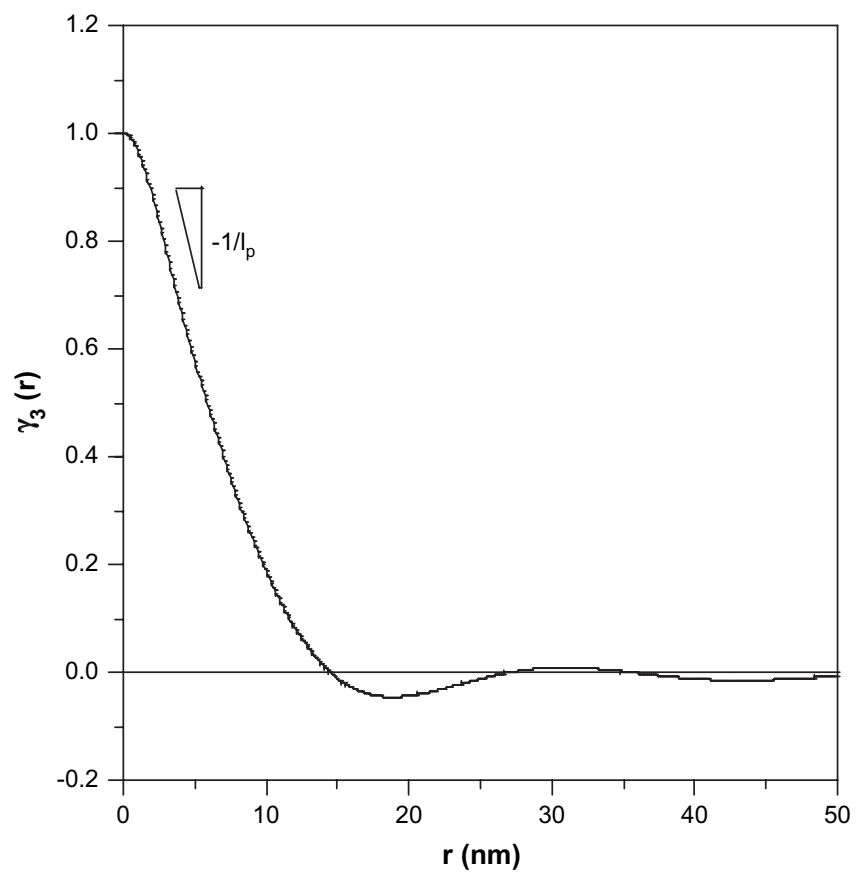

Fig. 8. The three-dimensional correlation function $\left(\gamma_{3}(r)\right)$ of HP. The Porod length $l_{\mathrm{p}}$ is determined from the slope of the linear region at small $r$.

where $\phi$ is the pore volume fraction and $(S / V)$ is the interfacial area per unit volume of the sample. In the case of spherical pores, $(S / V)=3 \phi / R$. Substituting into Eq. (5), we have

$\frac{1}{l_{\mathrm{p}}}=\frac{3}{4 R(1-\phi)}$

Again, assuming the spherical pores packed into a BCC lattice, substituting $\phi$ expressed by Eq. (1) into Eq. (6), we have

$\frac{1}{l_{\mathrm{p}}}=\frac{12}{D\left[8-\sqrt{3} \pi\left(\frac{D}{d}\right)^{3}\right]}$

Therefore, $D$ can be calculated from the value of $l_{\mathrm{p}}$ determined from the slope of the linear region of $\gamma_{3}(r)$ at small $r(r<5 \mathrm{~nm}$ in the present case). The value of $l_{\mathrm{p}}$ obtained from Fig. 8 was $11.9 \mathrm{~nm}$ and thus the radius of the pore was estimated to be $12.7 \mathrm{~nm}$. The pore volume fraction was hence calculated to be 0.30 using $R=12.7 \mathrm{~nm}$ and $d=33.5 \mathrm{~nm}$. The pore size, inter-domain distance and pore volume fraction evaluated from SAXS and TEM were quite consistent.

The morphological characterizations demonstrated that both LA and HA could serve as the structure-directing agent to produce nanoporous materials. The TEM observations revealed that the mesostructures templated from HA were more ordered than those derived from LA. In the precursor solutions both LA and HA should form well-ordered spherical micelles dispersed in the MSSQ matrix. The long-range order and the geometry of the micelles may, however, be perturbed by the chemical crosslinking of MSSQ at elevated temperatures. The solubilization of the polymer chains into the coronal regions causes stretching of the polymer chains and the coronal blocks, and hence a loss of conformational entropy. The 
stretching of the coronal blocks becomes more severe if the solubilized polymer chains have higher molecular weight; therefore, the solubilization of long polymer chains is entropically unfavorable. In the present system, the crosslinking of MSSQ for the preparation of the mesoporous material tends to produce network molecules of very large size. The solubilization of these molecules into the P2VP coronal region is thermodynamically unfavorable, such that a thermodynamic driving force should develop to expel these molecules out of the coronal regions, leading to a wet-to-dry brush transition. Such a wet-to-dry brush transition has actually been observed in the blends of PEO- $b$-PEP with epoxy [30], where the crosslinking of the epoxy during curing caused the epoxy networks to be expelled out of the PEO coronal regions in which the epoxy was originally uniformly solubilized (please see the schematic illustration from the original paper shown below). However, if the crosslinking reaction occurred much more rapidly than the structural reorganization processes (e.g. the diffusion of MSSQ out of the coronas, the reorganization of the junction points at the domain interface, etc.) involved in the wet-dry brush transition, the reaction may actually freeze the original domain structure. Consequently, the chemical crosslinking of MSSQ imposed two opposing effects on the morphological perturbation, namely, a thermodynamically prescribed wet-dry brush transition tending to transform the spherical domains to cylinders (or others with lower interfacial curvature) and a kinetic effect that trapped the original morphology if the reaction was sufficiently fast.

At the first stage of thermal treatment (i.e., drying at 80 and $100{ }^{\circ} \mathrm{C}$ sequentially for $30 \mathrm{~min}$ followed by baking at $120{ }^{\circ} \mathrm{C}$ for overnight) that yielded the hybrids, the crosslinking of MSSQ was minor, hence only the long-range order of the domains in the LA template was disturbed. Extensive crosslinking occurred in the subsequent slow heating to the calcination temperature for preparing the porous materials. In this case, the accompanied wet-dry brush was rapidly enough, such that a transformation of the domains morphology from sphere to cylinder took place effectively before the initial morphology was frozen by the crosslinking reaction. As a result, cylindrical pores were generated in $\mathbf{L P}$.

By contrast, the hetero-arm system exhibited a stronger resistance against the morphological perturbations in terms of domains' geometry and long-range order induced by the chemical crosslinking. This strong resistance should stem from the special molecular architecture of the copolymer, where three P2VP chains jointed to a common core slowed down the exclusion of the MSSQ out of the coronal regions since this diffusion should involve some cooperative motions with the P2VP blocks whose mobility became more restricted in HA. Moreover, the movement of the junction points at the domain interface, which was required for the domain structure transformation, was also slower than that in the linear counterpart, because each junction point here had to hold six polymer chains. Consequently, the initial micelle morphology was effectively fixed by the crosslinking reaction, leading to wellordered spherical pores in HP. The present study revealed that hetero-arm star copolymer served as a more effective template than its linear counterpart in the preparations of the mesoporous materials.

\section{Conclusions}

PS-P2VP block copolymers with linear or hetero-arm star architecture were used to prepare hybrid and mesoporous materials using EISA process. For the linear system, TEM observations revealed that the spherical domains in the hybrid transformed into cylindrical pores in the mesoporous material. We proposed that a wet-dry brush transition took place due to the extensive crosslinking of MSSQ on heating to the calcination temperature. This dewetting process was rapid enough, such that the micelle morphology transformed from sphere to cylinder before the initial morphology was frozen by the chemical crosslinking. By contrast, the hetero-arm system, in which three P2VP chains jointed to a common core slowed down the exclusion of the MSSQ out of the coronas, exhibited a stronger resistance against the morphological perturbation. In this case, the initial domain morphology was effectively fixed by the crosslinking reaction, leading to well-ordered spherical pores in the resultant mesoporous material. The present study demonstrated that hetero-arm star copolymer served as a more effective template than its linear counterpart in the preparations of MSSQ-based mesoporous materials.

\section{Acknowledgements}

This work was supported by the National Science Council of Taiwan under Contract No. NSC 94-2216-E002-025.

\section{References}

[1] Corma A. Chem Rev 1997;97:2373.

[2] Imhof A, Pine DJ. Nature 1997;389:948.

[3] Scott BJ, Wirnsberger G, Stucky GD. Chem Mater 2001;13:3140.

[4] Zhao XS, Su F, Yan Q, Guo W, Bao XY, Lv L, et al. J Mater Chem 2006; 16:637.

[5] Miller RD. Science 1999;286:421.

[6] Wang WC, Vora RH, Kang ET, Neoh KG, Ong CK, Chen LF. Adv Mater 2004; $16: 54$.

[7] Lee B, Oh W, Hwang Y, Park YH, Yoon J, Jin KS, et al. Adv Mater 2005; 17:696.

[8] Brinker CJ, Lu Y, Sellinger A, Fan H. Adv Mater 1999;11:579.

[9] Grosso D, Cagnol F, Soler-Illia GJ, Crepaldi EL, Amenitsch H, Brunet-Bruneau A, et al. Adv Funct Mater 2004;14:309.

[10] Ku Y, Gangull R, Drewlen CA, Anderson MT, Brinker CJ, Gong W, et al. Nature 1997;389:364.

[11] Doshi DA, Gibaud A, Goletto V, Lu MC, Gerung H, Ocko B, et al. J Am Chem Soc 2003;125:11646.

[12] Hillmyer H, Lipic PM, Hajduk DA, Almdal K, Bates FS. J Am Chem Soc 1997;119:2749.

[13] Göltner CG, Berton B, Krämer E, Antonietti M. Adv Mater 1999;11:395.

[14] Yu K, Smarsly B, Brinker CJ. Adv Funct Mater 2003;13:47.

[15] Lee J, Kim J, Lee Y, Yoon S, Oh SM, Hyeon T. Chem Mater 2004;16: 3323.

[16] Hampsey JE, Arsenault D, Hu Q, Lu Y. Chem Mater 2005;17:2475.

[17] Hadjichristidis N, Pispas S, Floudas G. Block copolymers: synthetic strategies, physical properties, and applications. New Jersey: WileyInterscience; 2003.

[18] Buzza DMA, Hamley IW, Fzea AH, Moniruzzaman M, Allgaier JB, Young RN, et al. Macromolecules 1999;32:7483. 
[19] Ishizu K, Uchida S. Prog Polym Sci 1999;24:1439.

[20] Zhu Y, Gido SP, Moshakou M, Iatrou H, Hadjichristidis N, Park S, et al. Macromolecules 2003;36:5719.

[21] Nandan B, Lee CH, Chen HL, Chen WC. Macromolecules 2005;38: 10117.

[22] Nandan B, Lee CH, Chen HL, Chen WC. Macromolecules 2006;39: 4460.

[23] Tsitsilianis C, Voulgaris D. Macromol Chem Phys 1997;198:997.

[24] Lee LH, Chen WC, Liu WC. J Polym Sci Part A Polym Chem 2001;40: 1560.
[25] Yang CC, Wu PT, Chen WC, Chen HL. Polymer 2004;45:5691.

[26] Chang Y, Chen CY, Chen WC. J Polym Sci Part B Polym Phys 2004;42: 4466.

[27] Matsushita Y, Mori K, Saguchi R, Nakao Y, Noda I, Nagasawa M. Macromolecules 1990;23:4313.

[28] Callister WD. Materials science and engineering. New York: John Wiley \& Sons; 1996 [chapter 2]

[29] Roe RJ. Methods of X-ray and neutron scattering in polymer science. New York: Oxford University Press; 2000 [chapter 5].

[30] Lipic PM, Bates FS, Hillmyer MA. J Am Chem Soc 1998;120:8963. 